\title{
Tribological Properties of Carbon Nanotube and Carbon Nanofiber Blended Polyvinylidene Fluoride Sheets Laminated on Steel Substrates
}

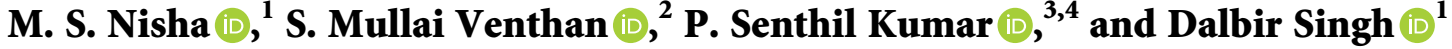 \\ ${ }^{1}$ School of Aeronautical Sciences, Hindustan Institute of Technology and Science, Chennai, India \\ ${ }^{2}$ Department of Mathematics, Rajalakshmi Engineering College, Chennai, India \\ ${ }^{3}$ Department of Chemical Engineering, Sri Sivasubramaniya Nadar College of Engineering, Chennai, India \\ ${ }^{4}$ Centre of Excellence in Water Research (CEWAR), Sri Sivasubramaniya Nadar College of Engineering, Chennai, India \\ Correspondence should be addressed to M. S. Nisha; msnisha@hindustanuniv.ac.in and P. Senthil Kumar; \\ senthilkumarp@ssn.edu.in
}

Received 17 October 2021; Revised 27 November 2021; Accepted 20 December 2021; Published 11 January 2022

Academic Editor: Vikranth Kumar Surasani

Copyright (c) 2022 M. S. Nisha et al. This is an open access article distributed under the Creative Commons Attribution License, which permits unrestricted use, distribution, and reproduction in any medium, provided the original work is properly cited.

\begin{abstract}
Nanostructured carbon dispersed polymer nanocomposites are promising materials for tribological applications. Carbon nanofiber $(\mathrm{CNF})$ and carbon nanotube (CNT) dispersed polyvinylidene fluoride (PVDF) nanocomposite was prepared by chemical synthesis route. Morphology and microstructure of well-dispersed CNF and CNT in PVDF were specified by scanning electron microscope and X-ray diffraction, respectively. Moreover, chemical and functional characteristics were examined by Raman spectroscopy and FTIR investigation. The friction coefficient of PVDF nanocomposite laminated on steel substrate decreased with an increase in the dispersed quantity of CNF and CNT. The friction coefficient of PVDF is approximately 0.27 ; however, the addition of carbon nanomaterial in PVDF will further decrease the friction coefficient between 0.24 and 0.17 . This value was significantly less in CNT dispersed PVDF nanocomposite. This could be explained by easy shearing and rolling action contact interfaces.
\end{abstract}

\section{Introduction}

Carbon has an extensive application in tribology for several years due to its corrosion resistive property, thermal stability, and outstanding mechanical behavior. Carbon materials which include fullerenes, CNT, CNF, graphene, and nanodiamond have incredible electrochemical properties. Moreover, they consist of both $\mathrm{sp}^{2}$ and $\mathrm{sp}^{3}$ carbon atoms which play a major role in instability [1]. Recently, power economization is an important topic in research progress, and still, it is a challenge in engineering application. Diana et al. have reported that nearly one-third of fuel consumption in the automobile industry is spent towards overcoming friction [2]. The natural consequences of tribological contacts are friction, frictional heat, and wear, and these consequences could be reduced by the incorporation of solid additives of low shear phase in bulk materials [3].
Thereby, tribology optimization and improvement are an important strategy for reduction in fuel consumption.

The lubrication behaviors of $\mathrm{C}_{60}$ and $\mathrm{C}_{70}$ fullerenes were extremely great due to their spherical structure and strong intramolecular and weak intermolecular bonding [4]. Further, Bhushan et al. [5, 6] investigated the hybrid method using fullerenes-silicon-coated substant to understand the friction and wear property. Subsequently, the results revealed that they provide a low friction coefficient. The addition of carbonous materials to metal significantly decreased the friction coefficient and wear rate [7]. Furthermore, metal matrix composite has been embedded with carbon fiber or graphite which resulted in the decrement of friction coefficient and performs as a self-lubricant [8].

For the past two decades, extensive researches have been carried out on polymer matrix composite reinforced with graphene. Almost all the outcomes show that the inclusion 
of graphene in the composites tremendously improves its property $[9,10]$. The shear resistance between the layers of graphene planes is weak, which slides easily under the tribological contacts helping to reduce the friction of graphene blended polymer [11]. Polymer composites possess copious strength-to-weight proportions and pliability in material pattern [12], in which the physical attributes could be adjusted for satiating the high wear resistance and low friction.

References [13-15] are studies conducted to analyze the tribological performance of carbon nanocomposites. The friction coefficient of 1.0 wt \% of $\mathrm{CNT}-\mathrm{Al}_{2} \mathrm{O}_{3}$ was achieved as $0.45-0.49$ [13], $1.0 \mathrm{wt} \%$ of MWCNT with polyethylene oxide reached to 0.28 frictional coefficient [14], and CNT with polystyrene's frictional coefficient was around 0.3 and graphene/SiC has been synthesized by attrition milling and hot isostatic pressing. Consequently, the frictional coefficient achieved was 0.396 [15].

Polymer is primarily used for several components which have been subjected to various advantages such as corrosion resistance, low friction, and vibration damping. Nevertheless, its thermal resistance and load carrying capability are feeble compared to metal and ceramics. To improve these properties, nano additives such as carbon black, carbon nanofiber (CNF), carbon nanotube (CNT), or graphene need to be included in the polymer for displaying exceptional mechanical, electrical, and thermal attributes [16]. Moreover, it is known that CNF and CNT are useful solid additives to enhance the tribological attributes of the polymer $[17,18]$. In the present study, functional crystalline PVDF is used to embed with solid micro/ nanofillers. This could be useful for improving their functional properties suitable for engineering applications [19]. It is reported that carbon-based nanofillers such as carbon black [20], CNTs [21], graphene, and graphene oxide [22] are suitable candidates with PVDF blending for the improvement of electrical conductivity, thermal conductivity, and dielectric constant of the material [23]. Moreover, such composite itself has self-lubricating properties which reduce the friction and guarantee security contra damage and distortion [24]. Microand nanoscale particle size of the carbon-based solid additives can easily enter between the sliding surfaces and prevent the direct asperity contact for the reduction of friction [25]. These additives have high compressive strength which may provide high wear resistance. The improvement in static and dynamic friction, scratch resistance, and sliding wear was determined by varying the amount of carbon black blended with PVDF and polyethylene [26].

In this work, tribological attributes of PVDF with the inclusion of different percentages of CNF and CNT have been investigated. The chemical structure and functional groups of PVDF/CNF and PVDF/CNT were determined by Raman spectroscopy and FTIR analysis. The friction behavior of these composites has been correlated with chemical structure and functionalities.

\section{Experimental Study}

2.1. Material Synthesis. PVDF of molecular weight $\sim 180,000$ with multiwall carbon nanotube (MWCNT) and carbon nanofiber (CNF) was used for synthesizing the PVDF/CNT and PVDF/CNF thin sheets. The outer diameter of the chemical vapor deposited MWCNT was 6-13 nm with an extent of $2.5-20 \mu \mathrm{m}$ and chemical pureness was ${ }^{2} 99 \%$ for synthesizing the PVDF/CNT composite sheets. Moreover, iron-free graphitized CNF, composed of conical platelets with a diameter of $100 \mathrm{~nm}$ and length $20-200 \mu \mathrm{m}$, was used for synthesizing the PVDF/CNF sheets. DMSO (dimethyl sulfoxide) with pureness of $\geq 99 \%$ was used as a solvent for the PVDF pellet. Further, SDS (sodium dodecyl sulfate) and acetone were used to solubilize the solution. Further, PVDF pellets were dissolved in the DMSO at $70^{\circ} \mathrm{C}$ using a magnetic stirrer at $60 \mathrm{rpm}$ for 30 minutes and fillers (CNT/CNF) were dissolved by acetone using a magnetic stirrer under room temperature with $60 \mathrm{rpm}$ for 30 minutes in a covered bottle to avoid evaporation. The solution was sonicated for one hour to break the particles, and during sonication, the SDS was added to stabilize the fillers. By using the electrospinning technique, the thin sheets were obtained from the prepared solution. The ultra-fine thin sheet was obtained on the surface of the collector drum with the applied voltage of 26 KVolt. The dimension of the fabricated thin sheet is $100 \mathrm{~mm} \times 100 \mathrm{~mm}$ with a thickness of $0.14 \mathrm{~mm}$, as shown in Figure 1. All the above materials were supplied by Sigma Aldrich.

2.2. Material Characterization. The chemical structure of the PVDF/CNF and PVDF/CNT with different wt \% was examined by a micro-Raman spectrometer (Andor SR-500i-C$\mathrm{R}, \lambda=532 \mathrm{~nm}$ ) in room temperature and ambient pressure. The friction and wear behaviors of PVDF/CNF and PVDF/ CNT sheets were calculated by ball-on-disc tribometer (Anton Paar, Switzerland), functioning in a linear-reciprocating manner. This was carried out by forming physical lamination of PVDF/CNF and PVDF/CNT sheets on diamond polished steel $(316 \mathrm{LN})$ substrates. The roughness of steel was ignored due to sheets lamination. Tribology test criteria such as normal load, sliding speed, and length of linear track were maintained; i.e., $1 \mathrm{~N}, 2 \mathrm{~cm} / \mathrm{s}$, and $4 \mathrm{~mm}$, accordingly. The tribology tests of these sheets were performed at room temperature and ambient pressure sliding against steel (100 Cr6) ball of $6 \mathrm{~mm}$ diameter with RMS roughness $R_{\mathrm{q}} \sim 45 \mathrm{~nm}$.

\section{Results and Discussion}

SEM images of PVDF nano-thin sheet doped with CNF and CNT are shown in Figures 2(a) and 2(b), respectively. The images show a uniform distribution of liner grains in CNF doped PVDF, and this gives rise to the high conductivity of the sample [26]. SEM images show that CNT is well dispersed in PVDF, and it is homogeneous and agglomerationfree.

Figure 3 shows the X-ray diffractograms of PVDF/CNF and PVDF/CNT nanocomposite, and the peaks are characteristic of $\beta$-phase [27]. The intensity of the peaks is enhanced with concentration.

Broadbands of $D$ and $G$ peaks, around $1340-1360 \mathrm{~cm}^{-1}$ and $1540-1580 \mathrm{~cm}^{-1}$, accordingly are illustrated in PVDF/ 


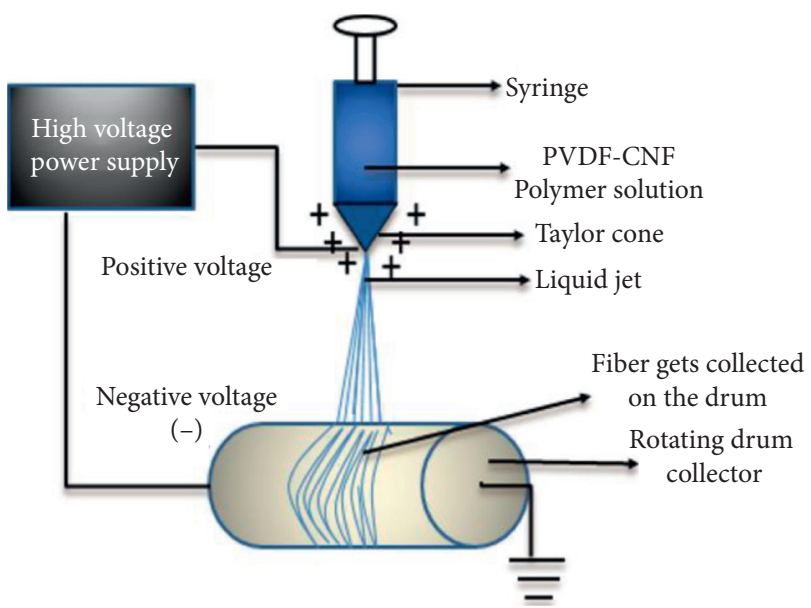

(a)

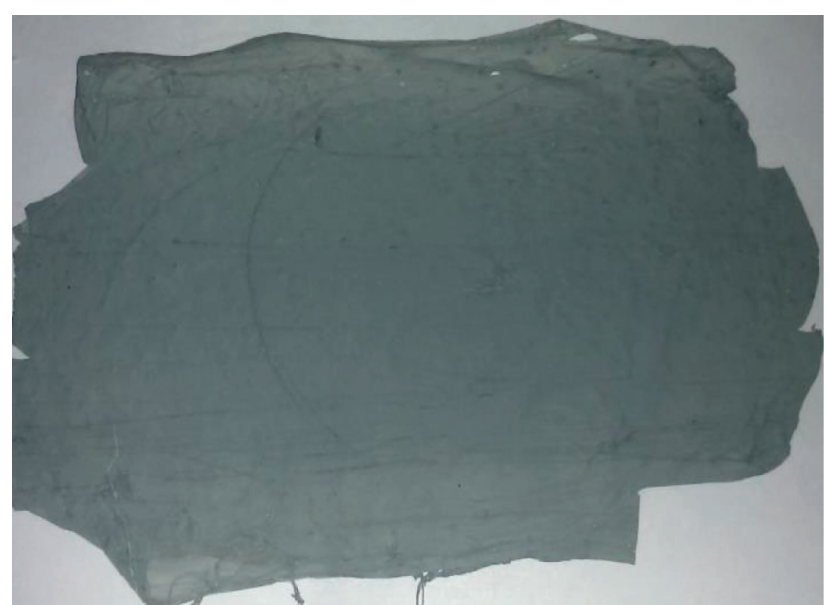

(b)

Figure 1: (a) Schematic diagram of thin fiber sheet fabrication. (b) Fabricated thin fiber sheet using electrospinning.

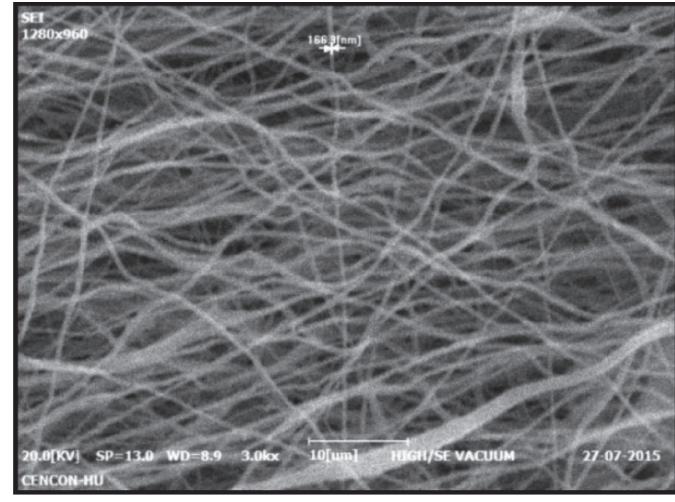

(a)

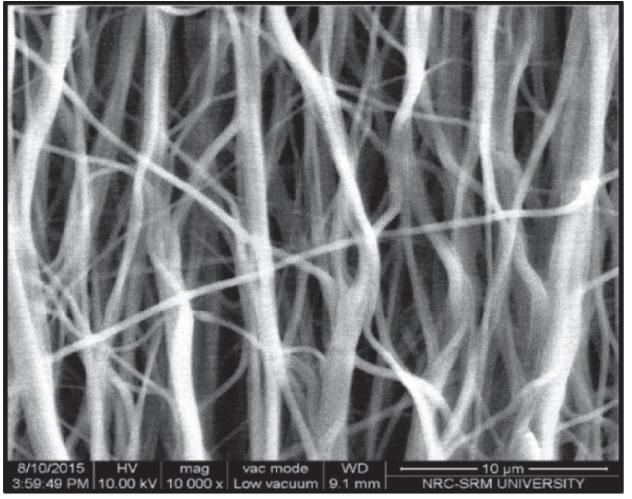

(b)

Figure 2: SEM image of (a) CNF and (b) CNT dispersed PVDF.

CNF samples (Figure 4). The intensity and the shape of these bands are enhanced in $0.16 \mathrm{wt} \% \mathrm{PVDF} / \mathrm{CNF}$ sample. In $\mathrm{PVDF} / \mathrm{CNT}$, these bands are centered on $1350 \mathrm{~cm}^{-1}$ and $1580 \mathrm{~cm}^{-1}$ which indicate vibrational spectra of CNT. The G-band is an inherent attribute of carbon nanotubes concerning vibrations in all $\mathrm{sp}^{2}$ carbon materials [27]. The foremost feature of the G-band is the attribute to Raman line shape.

The twain chief graphite bands exist in the Raman spectrum of MWCNT bundles in PVDF. The band at $1580 \mathrm{~cm}^{-1}$ ( $\mathrm{G}$ band) bestowed to the in-plane vibration of the $\mathrm{C}-\mathrm{C}$ bond. The $\mathrm{G}$ band is a tangential shear manner of carbon atoms that appertains to the stretching manner in the graphite plane [27]. The band at $1341 \mathrm{~cm}^{-1}$ (D band) is stimulated by the existence of disorderliness in carbon schemes. In PVDF/CNT, the $\mathrm{sp}^{2}$ phase is ordered; however, the Raman peak showed that PVDF/CNF is made by an amorphous carbon structure. PVDF modes are noticed at $630 \mathrm{~cm}^{-1}$ and $840 \mathrm{~cm}^{-1}$, and these are attributed to $\mathrm{CF}_{2}$ vibration [28]. A band at $840 \mathrm{~cm}^{-1}$ is visible because of the out of phase of $\mathrm{CH}_{2}$ rocking and $\mathrm{CF}_{2}$ stretching manner.
These functions are chiefly observed due to the $\alpha$-phase of PVDF and are normal for the all-trans arrangement of the PVDF chains. All-trans arrangements are ascribed to the injury caused by inflammation in the amorphous areas. These bands are more enhanced in PVDF/CNT composite. This is clear evidence of chemical linkage between PVDF and CNT/CNF.

In the FTIR spectra, the $\beta$-phase of PVDF in PVDF/CNF is characterized by peaks at $840,880,1175$, and $1276 \mathrm{~cm}^{-1}$ (Figure 5). An intense band at $1402 \mathrm{~cm}^{-1}$ agrees with the stretching vibration of $\mathrm{C}-\mathrm{H}$ bonds when the bands positioned at $1276 \mathrm{~cm}^{-1}$ and $1174 \mathrm{~cm}^{-1}$ are ascribed to the vibration of C-F bonds [29].

These peaks are slightly shifted to higher and lower frequencies as compared to neat PVDF which indicates chemical linkage with CNF. This is well evidenced by strong bands of stretching modes of alkyl moieties formed at $2855 \mathrm{~cm}^{-1}$ (symmetric) and $2917 \mathrm{~cm}^{-1}$ (asymmetric) [29]. This belongs to methylene groups $\left(-\mathrm{CH}_{2}-\right)$ of PVDF. However, PVDF bands are highly shifted in PVDF/CNT composite which indicates chemical intercalation of PVDF 


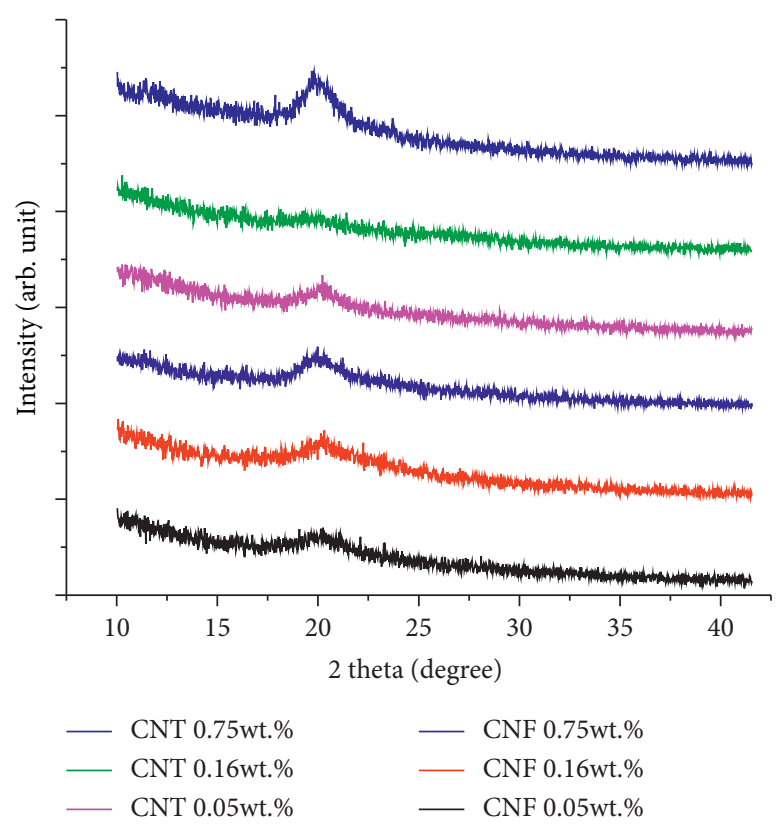

FIgure 3: XRD spectra of (a) CNF and (b) CNT dispersed PVDF.

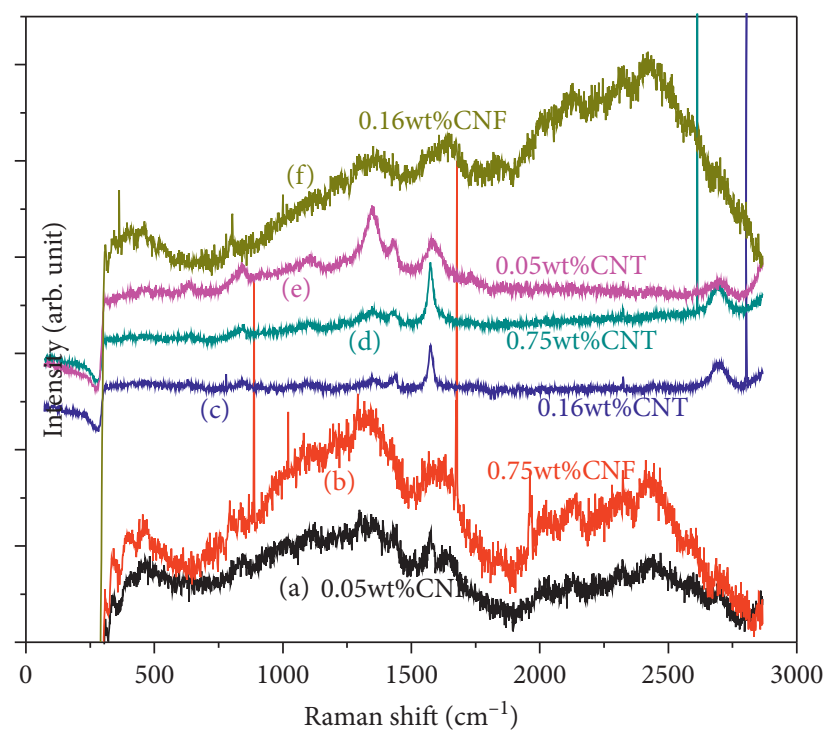

FIGURE 4: Raman spectra of PVDF/CNF and PVDF/CNT with various wt\%.

with CNT. A strong peak in PVDF/CNT appears at $3270 \mathrm{~cm}^{-1}$, which is ascribed to the stretching vibration of hydroxyl $(\mathrm{O}-\mathrm{H})$, indicating adsorption of water and moisture in the composite [30].

3.1. Friction Properties. The value of the friction coefficient of neat PVDF is approximately 0.27 . However, the friction coefficient of PVDF/CNT and PVDF/CNF is decreasing with an increase in CNT and CNF concentration, as shown in Figure 6. Moreover, this value is lower in PVDF/CNT nanocomposite.
This could be explained by an increase in the ordered $\mathrm{sp}^{2}$ phase in PVDF/CNT nanocomposite. This is well observed in Raman spectra. Ordered $\mathrm{sp}^{2}$ phase acts as an effective solid lubricant because of frail van der Waals forces acting betwixt the covalently bonded $\mathrm{sp}^{2}$ phase in the MWCNT structure. A weakly bonded $\mathrm{sp}^{2}$ plane easily shears and provides a low sliding friction coefficient [30].

Moreover, MWCNT rolls inside the contact which gives low resistance to rolling friction. Rolling and sliding resistance could be comparatively high in PVDF/CNF nanocomposite due to the absence of tube-like morphology and less ordered $\mathrm{sp}^{2}$ phase, respectively. Moreover, strong 


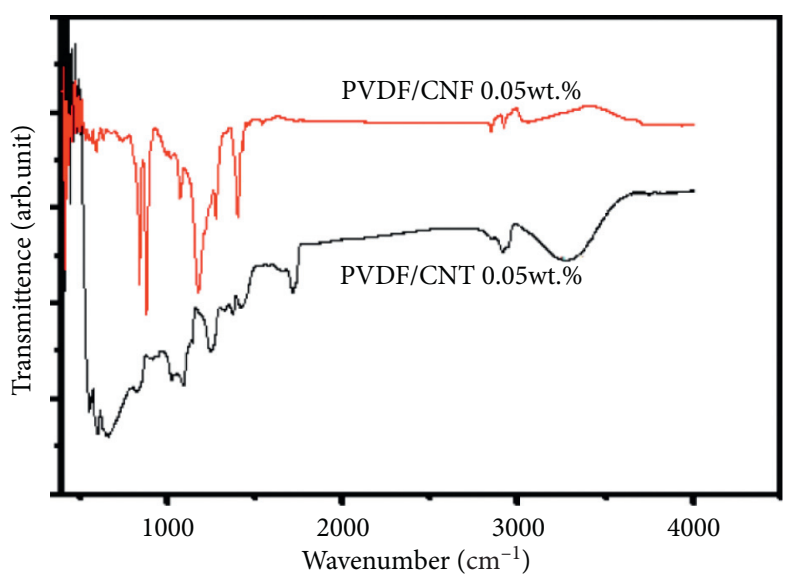

FIGURE 5: FTIR of PVDF/CNF and PVDF/CNT composite.

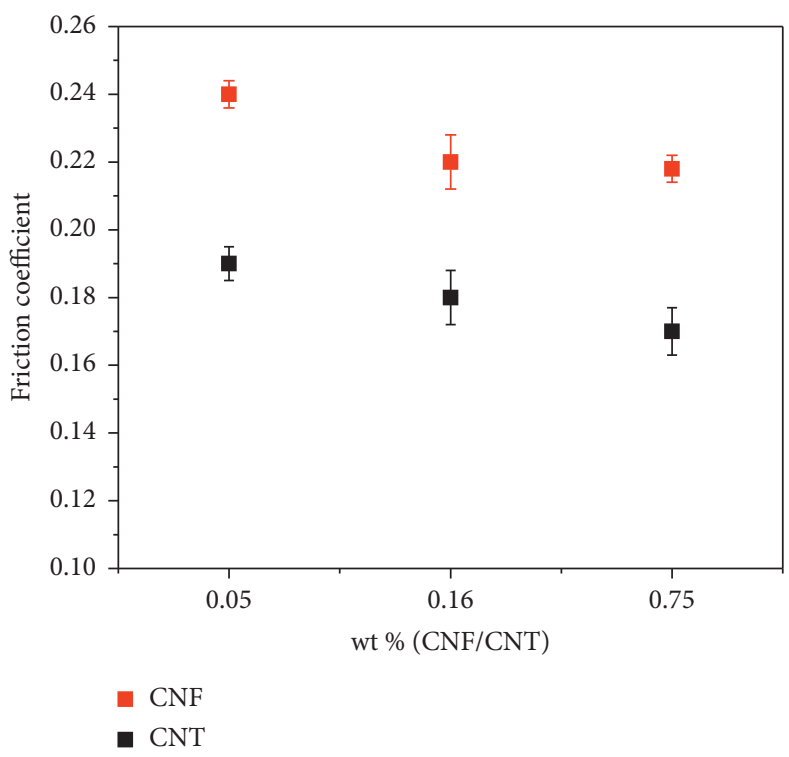

Figure 6: Tribological properties of PVDF/CNF and PVDF/CNT with various wt\%: load: $1 \mathrm{~N}$, sliding speed: $2 \mathrm{~cm} / \mathrm{s}$ (linear speed), ball: steel (100Cr6, diameter $6 \mathrm{~mm}$ ), sliding cycles: 10000, and condition: room temperature and ambient pressure.

intercalation of PVDF with CNT could be an important reason for low friction. This is well studied by FTIR analysis. Wear resistance of the PVDF/CNF and PVDF/CNT is found to be extremely high and no wear was observed in the optical image, as shown in Figure 6. This could be explained by improvement in load-bearing capacity which is provided by the combination of flexible properties of the polymer and low sharable characteristics of CNF and CNT.

\section{Conclusions}

CNF and CNT dispersed PVDF nanocomposite was prepared by chemical synthesis for tribological investigation. Well-grown and well-dispersed CNF and CNT were obtained at optimized conditions. Characteristic $\beta$-phase of PVDF was obtained in XRD analysis. Raman spectroscopy showed characteristic Raman bands which confirmed the dispersal of CNT and CNF in PVDF. The functional qualities and linkage of CNF and CNT were well established with PVDF. The friction coefficient of PVDF was decreased with an increase in the dispersed quantity of CNF and CNT. The value of friction coefficient was significantly less in CNT dispersed PVDF which is associated with the rolling mechanism of contact interfaces. The content of CNF indeed has a better lubrication effect on PVDF polymer which significantly reduces the friction coefficient.

\section{Data Availability}

The experimental and characterization data used to support the findings of this study are included within the article.

\section{Conflicts of Interest}

The authors declare that they have no conflicts of interest. 


\section{Acknowledgments}

The authors thank Dr. Niranjan Kumar, Institute for Physics of Microstructures, RAS, Russia, for the support.

\section{References}

[1] V. N. Mochalin, O. Shenderova, D. Ho, and Y. Gogotsi, "The properties and applications of nanodiamonds," Nature Nanotechnology, vol. 7, no. 1, pp. 11-23, 2012.

[2] B. Diana, S. A. Deshmukh, E. Ali, and A. V. Sumant, "Friction. Macroscale superlubricity enabled by graphene nanoscroll formation," Science, vol. 348, pp. 1118-1122, 2015.

[3] W. J. Bartz, "Tribology, lubricants and lubrication engineering - a review," Wear, vol. 49, no. 1, pp. 1-18, 1978.

[4] H. W. Kroto, "JR health, SC O'brien, RF curl, and RE smalley," Nature, vol. 318, no. 162, I985 pages, 1985.

[5] W. Krätschmer, L. D. Lamb, K. H. D. R. Fostiropoulos, and D. R. Huffman, "Solid C 60: a new form of carbon," Nature, vol. 347, no. 6291, pp. 354-358, 1990.

[6] B. Bhushan, B. K. Gupta, G. W. Van Cleef, C. Capp, and J. V. Coe, "Fullerene (C60) films for solid lubrication," Tribology Transactions, vol. 36, no. 4, pp. 573-580, 1993.

[7] E. Santos Júnior, S. d. S. Camargo Júnior, G. d. A. Soares, and M.-P. Delplancke-Ogletree, "Mechanical and tribological properties of Ti-containing carbon nanocomposite coatings deposited on TiAlV alloys," Materials Research, vol. 13, no. 4, pp. 527-533, 2010.

[8] X.-B. Liu, H.-Q. Liu, Y.-F. Liu et al., "Effects of temperature and normal load on tribological behavior of nickel-based high temperature self-lubricating wear-resistant composite coating," Composites Part B: Engineering, vol. 53, pp. 347-354, 2013.

[9] Z. Tang, Y. Lei, B. Guo, L. Zhang, and D. Jia, "The use of rhodamine B-decorated graphene as a reinforcement in polyvinyl alcohol composites," Polymer, vol. 53, no. 2, pp. 673-680, 2012.

[10] J. R. Potts, D. R. Dreyer, C. W. Bielawski, and R. S. Ruoff, "Graphene-based polymer nanocomposites," Polymer, vol. 52, no. 1, pp. 5-25, 2011.

[11] G. Pan, Q. Guo, J. Ding, W. Zhang, and X. Wang, "Tribological behaviors of graphite/epoxy two-phase composite coatings," Tribology International, vol. 43, no. 8 , pp. 1318-1325, 2010.

[12] A. P. Mouritz, E. Gellert, P. Burchill, and K. Challis, "Review of advanced composite structures for naval ships and submarines," Composite Structures, vol. 53, no. 1, pp. 21-42, 2001.

[13] A. K. Keshri, J. Huang, V. Singh, W. Choi, S. Seal, and A. Agarwal, "Synthesis of aluminum oxide coating with carbon nanotube reinforcement produced by chemical vapor deposition for improved fracture and wear resistance," Carbon, vol. 48, no. 2, pp. 431-442, 2010.

[14] B.-H. Ryu, A. J. Barthel, H.-J. Kim et al., "Tribological properties of carbon nanotube-polyethylene oxide composite coatings," Composites Science and Technology, vol. 101, pp. 102-109, 2014.

[15] S. C. Tjong, "Recent progress in the development and properties of novel metal matrix nanocomposites reinforced with carbon nanotubes and graphene nanosheets," Materials Science and Engineering: R: Reports, vol. 74, no. 10, pp. 281-350, 2013.

[16] Z. Spitalsky, D. Tasis, K. Papagelis, and C. Galiotis, "Carbon nanotube-polymer composites: chemistry, processing, mechanical and electrical properties," Progress in Polymer Science, vol. 35, no. 3, pp. 357-401, 2010.

[17] B. Dong, Z. Yang, Y. Huang, and H. L. Li, "Study on tribological properties of multi-walled carbon nanotubes/epoxy resin nanocomposites," Tribology Letters, vol. 20, no. 3, pp. 251-254, 2005.

[18] J. K. Katiyar, S. K. Sinha, and A. Kumar, "Effects of carbon fillers on the tribological and mechanical properties of an epoxy-based polymer (SU-8)," Tribology: Materials, Surfaces \& Interfaces, vol. 10, no. 1, pp. 33-44, 2016.

[19] M. Benz, W. B. Euler, and O. J. Gregory, "The role of solution phase water on the deposition of thin films of poly(vinylidene fluoride)," Macromolecules, vol. 35, no. 7, pp. 2682-2688, 2002.

[20] Y. Luo, W. Yu, and F. Xu, "Surface modification and vaporinduced response of poly(vinylidene fluoride)/carbon black composite conductive thin films," Polymer - Plastics Technology and Engineering, vol. 50, no. 11, pp. 1084-1090, 2011.

[21] Q. Liu, J. Tu, X. Wang, W. Yu, W. Zheng, and Z. Zhao, "Electrical conductivity of carbon nanotube/poly(vinylidene fluoride) composites prepared by high-speed mechanical mixing," Carbon, vol. 50, no. 1, pp. 339-341, 2012.

[22] V. Eswaraiah, K. Balasubramaniam, and S. Ramaprabhu, "Functionalized graphene reinforced thermoplastic nanocomposites as strain sensors in structural health monitoring," Journal of Materials Chemistry, vol. 21, no. 34, pp. 1262612628, 2011.

[23] M. El Achaby, F. Z. Arrakhiz, S. Vaudreuil, E. M. Essassi, A. Qaiss, and M. Bousmina, "Preparation and characterization of melt-blended graphene nanosheets-poly(vinylidene fluoride) nanocomposites with enhanced properties," Journal of Applied Polymer Science, vol. 127, no. 6, pp. 4697-4707, 2013.

[24] D. Berman, A. Erdemir, A. V. Zinovev, and A. V. Sumant, "Nanoscale friction properties of graphene and graphene oxide," Diamond and Related Materials, vol. 54, pp. 91-96, 2015.

[25] M. Campo, A. Jiménez-Suárez, and A. Ureña, "Effect of type, percentage and dispersion method of multi-walled carbon nanotubes on tribological properties of epoxy composites," Wear, vol. 324-325, pp. 100-108, 2015.

[26] W. Brostow, M. Keselman, I. Mironi-Harpaz, M. Narkis, and R. Peirce, "Effects of carbon black on tribology of blends of poly(vinylidene fluoride) with irradiated and non-irradiated ultrahigh molecular weight polyethylene," Polymer, vol. 46, no. 14, pp. 5058-5064, 2005.

[27] A. C. Ferrari and J. Robertson, "Interpretation of Raman spectra of disordered and amorphous carbon," Physical Review B, vol. 61, no. 20, pp. 14095-14107, 2000.

[28] Y. Ji, J. Liu, Y. Jiang, and Y. Liu, "Analysis of Raman and infrared spectra of poly(vinylidene fluoride) irradiated by $\mathrm{KrF}$ excimer laser," Spectrochimica Acta Part A: Molecular and Biomolecular Spectroscopy, vol. 70, no. 2, pp. 297-300, 2008.

[29] P. Xu, W. Fu, Z. Cui, and Y. Ding, "Synergistic promotion of polar phase crystallization of PVDF by ionic liquid with PEG segment," Applied Surface Science, vol. 444, pp. 480-484, 2018.

[30] B. Gupta, N. Kumar, K. Panda, A. A. Melvin, S. Joshi, and S. Dash, "Molecular-pillar-supported functionalized reduced graphene-oxide for energy efficient lubrication," Advanced Materials Interfaces, vol. 3, no. 13, pp. 1600161-1600169, 2016. 